\title{
Exercise-induced gastrointestinal disturbances: potential amelioration with a ginger containing beverage
}

\author{
D. Ball ${ }^{1}$, G. Ashley ${ }^{2}$ and H. Stradling ${ }^{1}$ \\ ${ }^{1}$ School of Life Sciences, Heriot-Watt University, Edinburgh, EH14 $4 A S$ and ${ }^{2}$ Primerdesign Ltd, Southampton, \\ SO16 $0 \mathrm{AJ}$
}

The frequency of upper and lower gastrointestinal disturbance as a function of exercise is reported to be between 30 and $70 \%{ }^{(1)}$. With the severity of symptoms ranging from mild stomach discomfort to severe diarrhoea ${ }^{(1)}$ and a common observation that the consumption of beverages either before or during exercise may increase the incidence rate by over $25 \%{ }^{(2)}$. Recent reports suggest that ginger containing beverages can reduce the symptoms of nausea and vomiting when administered during chemotherapy ${ }^{(3)}$. The purpose of the present study was to examine the effects of consuming an isotonic beverage with and without ginger on the frequency and severity of gastrointestinal disturbance during and after exercise.

In accordance with the Declaration of Helsinki and Ethics committee approval 40 recreational athletes (23 male, 17 female) volunteered to participate. On three separate occasions, at least 7 days apart, each volunteer completed a $5 \mathrm{~km}$ run around the same course. Volunteers completed a 5 item questionnaire that assessed upper and lower gastrointestinal (GI) symptoms ${ }^{(4)}$ before and after exercise by placing a mark on $10 \mathrm{~cm}$ line rated as 0 (never) and 10 (always). Each volunteer consumed a total of $450 \mathrm{ml}$ of either water or beverage A or beverage B; $225 \mathrm{ml}$ immediately prior to and $225 \mathrm{ml}$ following exercise. Beverage A contained $7 \cdot 5 \%$ glucose, $10 \mathrm{mM} \mathrm{NaCl}$, citric acid, $\mathrm{K}$ sorbate and $62.5 \mathrm{ml}$ of ginger root extract per $1 \mathrm{~L}$; beverage B was identical to beverage A but the ginger was replaced with $62.5 \mathrm{ml}$ of carrot extract. Two further questionnaires were administered assessing beverage tolerance immediately and 30 minutes after exercise. Data were analysed using Wilcoxon Signed rank tests and are reported as the median (range).

The incidence of upper GI disturbance increased $(\mathrm{P}<0.05)$ as a function of exercise; stomach problems increased from pre-exercise $1 \cdot 7(0 \cdot 1-6 \cdot 3)$ to $2 \cdot 0(0 \cdot 1-8 \cdot 4)$ during exercise and nausea increased from pre-exercise $1 \cdot 1(0 \cdot 1-4 \cdot 5)$ to $2 \cdot 0(01-7 \cdot 6)$ during exercise. All other measures of GI disturbance were similar between pre- during and post-exercise. Consuming the beverages did not exacerbate the GI symptoms during exercise. However, after exercise the prevalence of stomach problems (4.6 (0.3-6.6)) and nausea (4.5 (0.3-9) decreased $(\mathrm{P}<0.05)$ with beverage A but neither beverage $\mathrm{B}$ nor water had this effect stomach problems $(5(0 \cdot 2-8 \cdot 2))$ nausea $(5(0 \cdot 2-7))$. Ginger administration during chemotherapy has been reported to reduce symptoms of nausea ${ }^{(3)}$ and these data support the observation that ginger has a similar effect following exercise. The exact mechanism behind this effect is unresolved but has been linked to the antagonist effects on serontonergic 5 HT receptors ${ }^{(3)}$.

1. Simons SM \& Shaskan GG (2005) Int Sports Med J 5, 162-170.

2. Peters H, Bos M, Seegbregts L et al. (1999) Am J Gastroenterol 94, 1570-1581.

3. Sontakke S, Thawani V \& Naik MS (2003) Indian J Pharma 35, 32-36.

4. Pfeiffer B, Stellignwerff T, Hodgson AB et al. (2012) Med Sci Sports Exerc 44, 344-351. 